\title{
Purposeless repeated acquisition time-lapse seismic data processing
}

\author{
Li Jingye ${ }^{1,2}$, Chen Xiaohong ${ }^{3 *}$, Zhao $\mathrm{Wei}^{4}$ and Zhang Yunpeng ${ }^{4}$ \\ ${ }^{1}$ Key Laboratory for Hydrocarbon Accumulation Mechanism, Ministry of Education, China University of Petroleum, \\ Beijing 102249, China \\ ${ }^{2}$ Basin \& Reservoir Research Center, China University of Petroleum, Beijing 102249, China \\ ${ }^{3}$ School of Resource and Information, China University of Petroleum, Beijing 102249, China \\ ${ }^{4}$ Research Center of CNOOC, Beijing 100027, China
}

\begin{abstract}
In China, most oil fields are continental sedimentation with strong heterogeneity, which on one side makes the reservoir prospecting and development more difficult, but on the other side provides more space for searching residual oil in matured fields. Time-lapse seismic reservoir monitoring technique is one of most important techniques to define residual oil distribution. According to the demand for and development of time-lapse seismic reservoir monitoring in China, purposeless repeated acquisition time-lapse seismic data processing was studied. The four key steps in purposeless repeated acquisition time-lapse seismic data processing, including amplitude-preserved processing with relative consistency, rebinning, match filtering and difference calculation, were analyzed by combining theory and real seismic data processing. Meanwhile, quality control during real time-lapse seismic processing was emphasized.
\end{abstract}

Key words: Time-lapse seismic, purposeless repeated acquisition, rebinning, match filtering, amplitudepreserved processing

\section{Introduction}

As a hot-point in the current study on reservoir geophysics, time-lapse seismic reservoir monitoring, which integrates geology, geophysics, petrophysics and reservoir engineering, realizes the transformation from static description to dynamic prediction of reservoir properties, quick reservoir evaluation, adjustment of oil field development scheme, and improvement of oil and gas recovery (Anno and Routh, 2007; Hong et al, 2007; Li and Chen, 2006; Wang, 1997). Shell, $\mathrm{BP}$, and other major oil companies and service companies have paid great attention to this technique (Kloosterman and Kelly, 2003; Andersen and van Wijngaarden, 2007). Based on different AVO responses for different reservoir parameters variations, Landro et al. have realized accurate description of reservoir parameters with the method of time-lapse seismic AVO (Amplitude versus offset)(Landro, 2001; Landro, et al, 2003). In recent years, with the expansion of experimental study in Xinjiang, Shengli, Liaohe Oilfields, time-lapse seismic reservoir monitoring has made great progress and achieved some success in China.

Under the fundamental assumption that seismic responses related to non-reservoir factors are consistent and those related to reservoir are variable with time during production, time-lapse seismic technique mainly uses differences between two 3D seismic surveys acquired at different times to analyze reservoir fluid variations. The aim of time-lapse seismic

*Corresponding author. email: chenxh@cup.edu.cn

Received October 20, 2006 data processing is to eliminate seismic responses related to reservoir static properties, such as structure, lithology, and other non-reservoir factors, and to retain responses related to dynamic fluid properties, such as fluid saturation, pressure and temperature. Purposeless repeated seismic data are repeated acquisition data for the same reservoir at different times, but not mainly for the purposes of time-lapse seismic study. On one side, time-lapse seismic process for purposeless repeated acquisition data is more difficult and requires more novel techniques due to acquisition designed not for time-lapse seismic study, but on the other side, the project expense is much less for saving the cost of seismic data acquisition, which makes the time-lapse seismic technique more acceptable and prompts its development in China (Yun et al, 1999). In the paper, the key techniques in time-lapse seismic data processing for purposeless repeated acquisition are studied, including amplitude-preserved processing with relative consistency, rebinning, match filtering and difference calculation, which may provide a guide for time-lapse seismic technique application in China.

\section{Amplitude-preserved processing with relative consistency}

Time-lapse seismic reservoir monitoring mainly uses seismic amplitude differences to monitor reservoir dynamic variations, which makes it necessary to preserve amplitude information with processing flow consistency to the maximum extent for two seismic data, and to keep amplitude consistent laterally and vertically for each data set. The main processing 
techniques contain seismic wavelet process, deconvolution, and quality analysis and quality control.

\subsection{Seismic wavelet processing}

Since purposeless repeated acquisition seismic data have great differences in geometry, instrument, acquisition parameters and environmental factors, there are huge differences between two data sets in wavelet, bandwidth and signal-to-noise ratio etc.(An and Wei, 2006), which brings lots of difficulty to time-lapse seismic data processing. Among these differences, seismic wavelet diversity is one of the main reasons, but fortunately this can be eliminated by wavelet processing. Based on the statistics of the wavelets near the target formation, inverse wavelet is designed to process the wavelets with the aim to make the processed wavelets consistent.

\subsection{Refined deconvolution}

As one of key steps in seismic data processing, deconvolution is used to improve seismic resolution by suppressing multiples and shortening wavelets. For the reason of minimum-phase assumption, it is necessary to conduct wavelet processing before deconvolution to keep seismic data in minimum phase. To further increase consistency between two data sets, deconvolution parameters are determined through precise analysis to attenuate reverberation, improve resolution and suppress multiples.

\subsection{Analysis during processing and quality control}

Due to the stricter requirements for time-lapse seismic processing than for conventional processing, it is necessary to eliminate seismic response variations caused by non-reservoir factors, and to avoid artifacts brought in during seismic processing as much as possible. Seismic data are analyzed after every step of processing, including frequency scanning, wavelet analysis, correlation analysis, amplitude monitoring laterally and vertically, and so on. Fig. $1 \mathrm{a}$ and $1 \mathrm{~b}$ respectively show frequency spectra after noise reduction and multiple attenuation. If amplitude relative variations are unchanged, and processing results are acceptable, the processing flow and parameters are reasonable for time-lapse seismic data processing. Otherwise, they will be further adjusted up to meeting processing requirement.

Fig. 2 shows the comparison between the results of conventional processing and amplitude-preserved processing with relative consistency. It demonstrates that amplitudepreserved processing with relative consistency does better in seismic data relativity-preservation for more geologic information kept but with lower $\mathrm{S} / \mathrm{N}$ rate.

\section{Rebinning}

The changes of surface installations, technical equipment and other factors at different acquisition times make it hard to acquire two data sets with same geometry and acquisition parameters. With 3D seismic data, it is mainly manifested as the different sizes and positions of reflection bins. To compare time-lapse data acquired at different times, the reflection
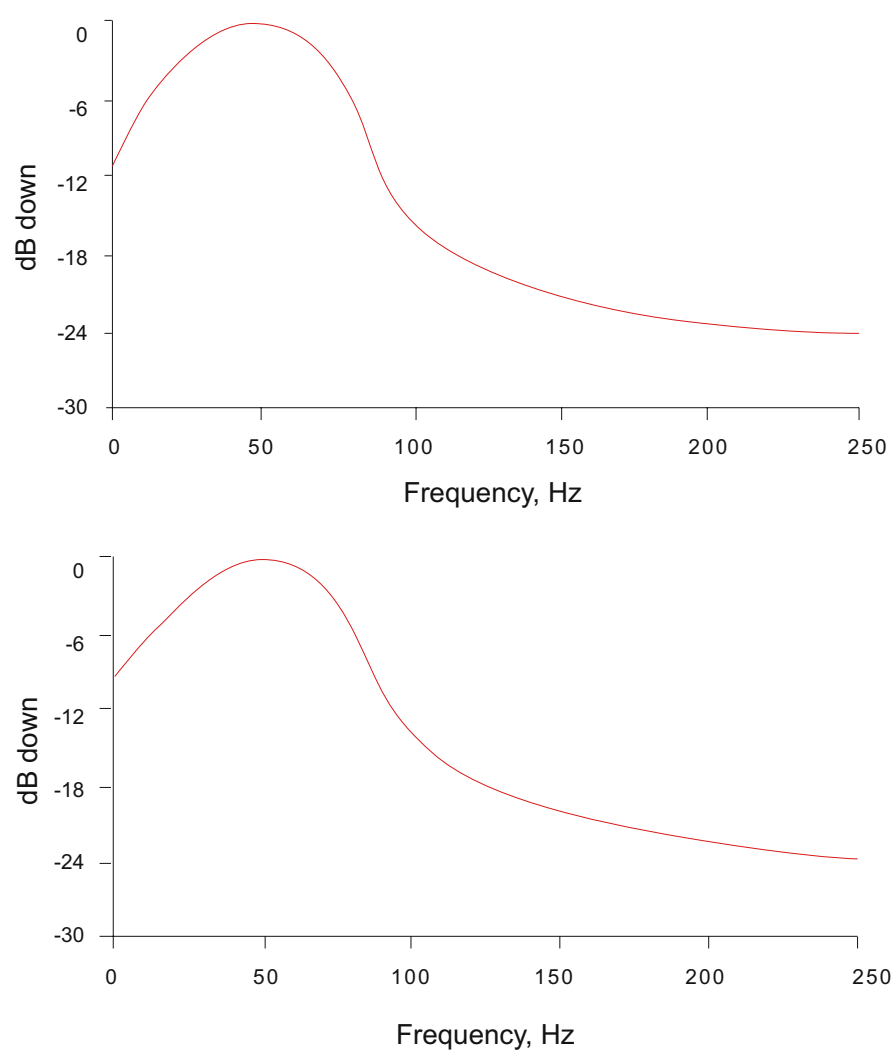

Fig. 1 Frequency spectrum monitor during process. (a) Frequency spectrum after noise reduction; (b) Frequency spectrum after multiple attenuation

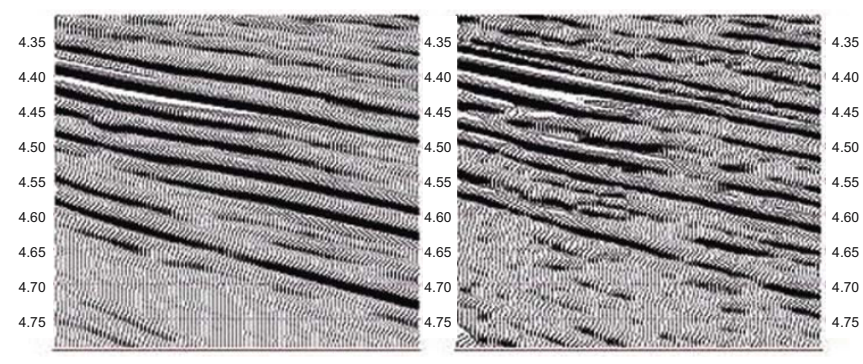

Fig. 2 Comparison of the seismic data processing results. (a) Seismic section by conventional processing flow; (b)Seismic section by amplitude-preserved processing flow with relative consistency

bins or points should be corrected to the same position, which is referred to as rebinning ( Lu et al, 2004). Rebinning processing can be conducted by many methods, including correlative traces extraction, linear interpolation, F-K interpolation, T-X dynamic difference interpolation, and so on. Each method with its own advantages and disadvantages is suitable for different situations, but their aims are all to make time-lapse seismic data more consistent while doing as little harm as possible to amplitude information.

\subsection{Principle of dynamic difference interpolation}

Based on the lateral coherence of seismic events, dynamic difference interpolation is conducted by weighting interpolation trace by trace, after an accurate time dip angle calculation for each point by the method of moving difference. According to the precise dip angle calculated by 
the dynamic difference method, multiple traces interpolation can be conducted by combining with the anti-distance weighting method when there is multi-trace gap in the seismic section. As an effective method with high precision and high speed for seismic data interpolation, the dynamic difference interpolation method can be applied to linear or nonlinear events, and equal interval or unequal interval interpolation.

The key step of the dynamic difference technique is to calculate correctly the time dip angle, which can be summarized as follows. Under the assumption that there are $M$ traces in a 2D seismic section, $N$ points in a trace, a difference gather can be obtained when the time dip angle is calculated for sample $j$ in trace $i+1$ by doing trace difference, during which the trace $i+1$ is kept still and trace $i$ moves by different time intervals $k(-1 \underline{k \leq 1)}$. Then, to sample $j$, the time dip angle is moving time corresponding to the minimum value in the difference gathers. The computation process of trace interpolation is as follows.

$$
\operatorname{diff}_{i+1, j}(k)=\left|x_{i+1}-x_{i+1, j}(k)\right|
$$

1) The absolute value of the two traces difference is calculated by the following equation.

Adding $k$ by 1 , the above step is repeated to obtain the difference gathers.

2) Autocorrelation for every trace within difference gathers in the small time window is averaged, and the result is given to the middle sample. The purpose of the step is to make the differences between samples larger when calculating the dip angle, which is convenient for doing comparison. In the same time, smoothing processing is conducted on the difference gathers to eliminate the noise effects on the comparison.

$\operatorname{diff}_{i+1, j}(k)=\frac{1}{L} \sum_{n=\frac{L}{2}}^{\frac{L}{2}} \operatorname{diff}_{i+1, j}(k) \cdot \operatorname{diff}_{i+1, j}(k) \quad k=-1,-1+1, \wedge, 1$

3) The dip angle of the sample $\mathrm{j}$ is calculated.

$$
\operatorname{dip}_{i+1, j}=\min \left\{\operatorname{dip}_{i+1, j}(-1+1), \wedge, \operatorname{diff}_{i+1, j}(1)\right\}
$$

Here $i$ is the trace number, $i=1,2, \ldots \mathrm{M}, j$ is the sample, $j=1,2, \ldots \mathrm{N} ; k$ is the auxiliary channel shift ranging from -1 to 1 , and 1 is the length of the small window, selected according to the real record, generally not more than half wavelet length. According to the amplitudes on both sides of the interpolated point along the dip angle calculated by the Equation (3), the interpolated point's amplitude is obtained by the Lagrange interpolation method.

Dynamic difference interpolation can be applied to irregular trace interpolation, for one trace or multiple traces interpolation between two traces, and for the data with spatial aliasing as well. Therefore it can meet the needs of time-lapse seismic rebinning processing. 3D interpolation is realized by 2D interpolation in $X$ and $Y$ direction respectively.

\subsection{Quality control of rebinning}

Rebinning processing makes the bins of the monitor data the same with those of the reference data, and the quality control of rebinning is necessary to verify the effectiveness of rebinning processing. There are two methods to test the effectiveness of rebinning. The first one is to analyze variations of wave form and frequency spectrum. Fig. 3 shows the frequency spectra variations before and after rebinning for the seismic data of $X$ Oil Field. The second one is to analyze quantitatively the effects of rebinning error on time-lapse seismic data processing, that is, rebinning the seismic data twice, comparing the result with the data before rebinning and analyzing the rebinning error. Fig. 4 shows RMS amplitude differences before rebinning and after rebinning twice and RMS amplitude before rebinning for the target formation. The quantitative analysis demonstrates that the seismic data error is less than $0.8 \%$ after rebinning twice, and the rebinning error can meet the demands of time-lapse seismic processing study.

\section{Time-lapse seismic matching processing}

Time-lapse seismic matching processing is a composite process, including match filtering, amplitude calibration, static time shift and other corrections (Chen, 1999; Gan et al, 2003). Matching processing should make the energy, time and phase of the two 3D seismic data trend consistent because there is no fluid variation to make the non-reservoir part of the two surveys acquired at different times vary with time.

\subsection{Principle of time-lapse seismic matching processing}

Matching processing is to eliminate the differences in time, amplitude and frequency of two time-lapse seismic data sets. According to the differences in time, amplitude frequency and phase aspects, multiple matching operators are applied during the correction. The matching operator is either a global filter, which is used for all the lines and traces, or a local filter, which locally corrects for the single trace of one line. Time-lapse seismic matching processing includes time shift correction, energy correction, frequency correction, phase correction, amplitude correction, structure correction, match filtering based on normal formation and so on.

1) Energy correction

Time-lapse seismic energy correction includes lateral energy equalization, which makes seismic energy distribute evenly and decreases the effects of uneven distribution on seismic differences by energy statistics and correction in a big window, and vertical energy matching processing, which realizes vertical energy equalization by amplitude envelope matching in a big window without effects on local difference analysis.

2) Time shift correction

Time shift correction is conducted by the method of cross correlation to calculate the time shift of the monitor data. The simplest case is that the time shift has only one value, that is, it is consistent in space and time. If every trace has only one time shift, the correction is variant in space and consistent in time. If each trace has different time shifts in different windows, it is variant in space and time, and time shifts at different samples can be interpolated. Purposeless repeated acquisition seismic data usually need a nonlinear time shift correction. 


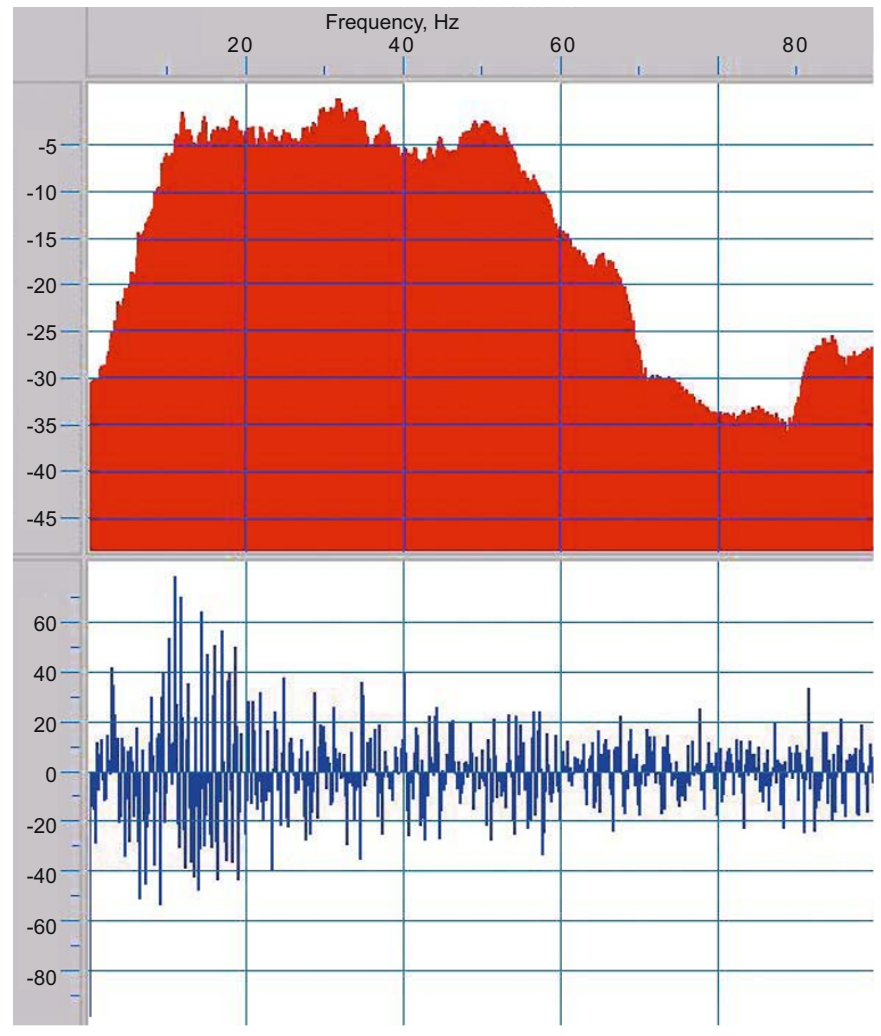

(a)Before rebinning

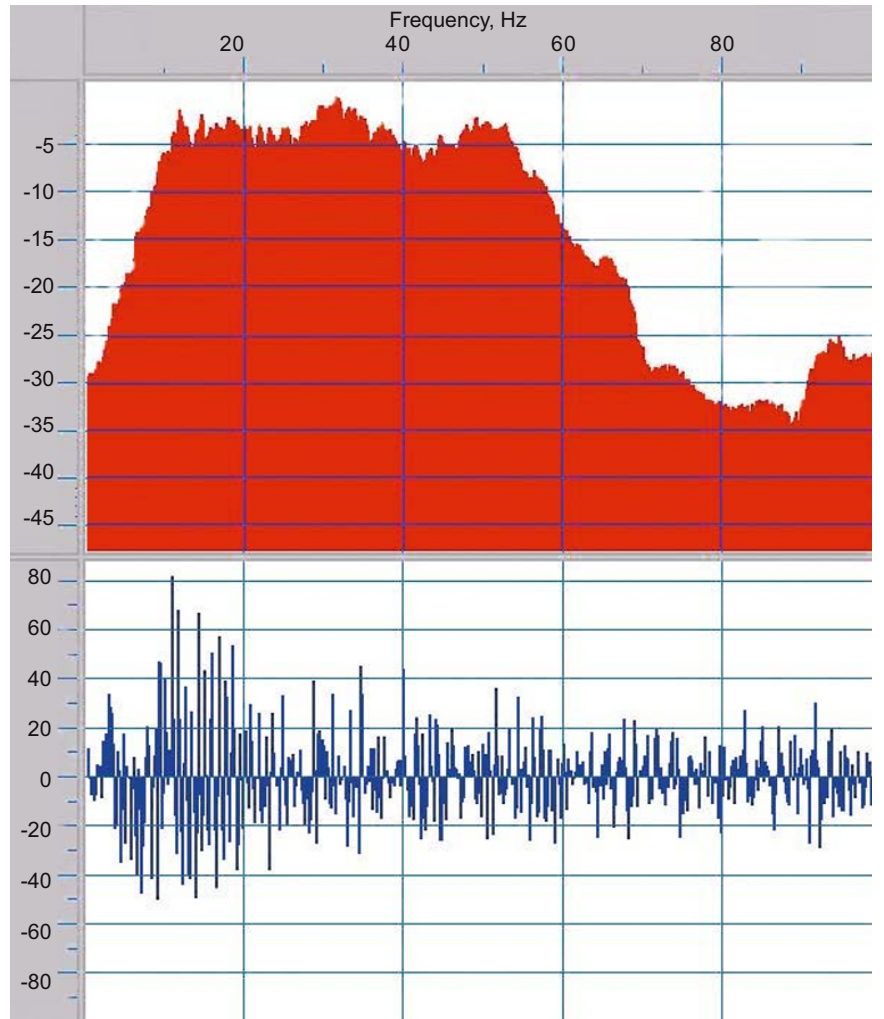

(b)After rebinning

Fig. 3 Comparison of frequency spectrum before and after rebinning

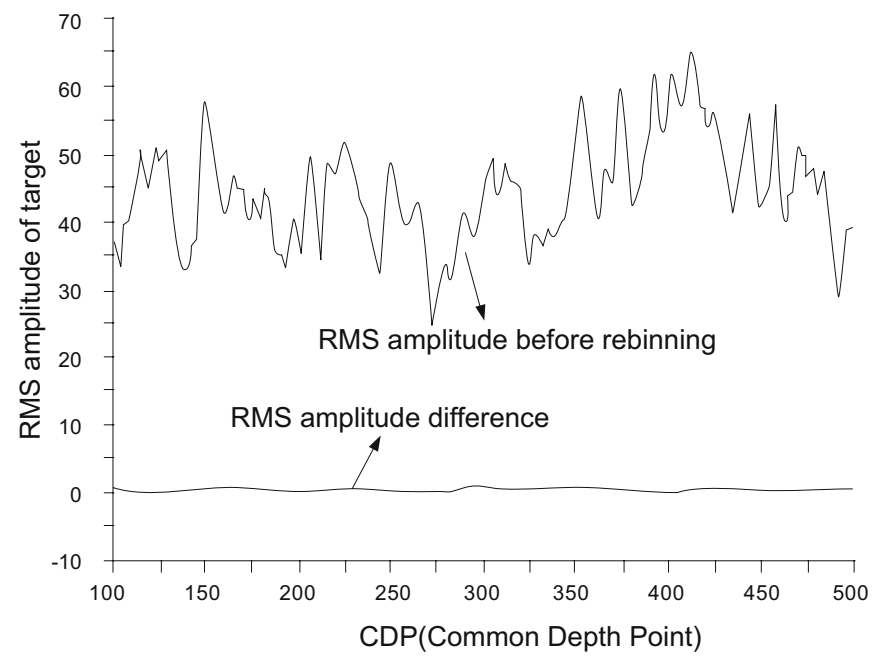

Fig. 4 RMS amplitude of target formation before rebinning and its comparison with that after rebinning twice

3) Frequency correction

Frequency correction firstly needs to conduct frequency spectrum analysis of the two data sets, and then to correct the monitor data basing on the smoothed frequency spectrum of the reference data. It is also realized by band-pass filtering, during which the lower frequency and narrower bandwidth should be taken as the reference for the two data sets.

4) Phase correction

Phase correction is to make the phase of the monitor data the same as that of the reference data. The simplest method is to correct the phases of the two seismic data sets to zero phase together. It is also achieved by designing the phase filter through phase scanning of the monitor datasets and comparing it with the reference data, and phase filtering of the monitor data.

5) Spatial position error correction

The differences in geometry and acquisition orientation of purposeless repeated acquisition may cause spatial position error for the same reflection point in different migrated sections. To achieve spatial error correction, the optimum shifts in $\mathrm{x}, \mathrm{y}$ and time directions are calculated by $3 \mathrm{D}$ crosscorrelation of small data volumes from the two data sets.

6) Match filtering based on normal formation

The non-reservoir parts of the two seismic data sets acquired at different times in the same area are identical theoretically, but in fact they differ significantly due to different acquisition conditions and other factors. To remove these differences, objective functional equation (4) should be minimized (Jin et al, 2003; Li and Hu, 2004).

Here, $D(t)$ is the difference between the two seismic data

$$
D(t)=\left\|S_{r e f}(t)-N S_{\text {mon }}(t)\right\|
$$

sets, $\mathrm{S}_{\text {re }}(t)$ is the reference seismic record, $\mathrm{S}_{\text {mon }}(t)$ is the monitor seismic record, and $\mathrm{N}$ is the designed operator. Applying the designer operator to the whole seismic trace can eliminate the unreasonable differences caused by acquisition conditions and other factors, which is called matching processing based on the normal formation. The normal formation should be stable in the whole area, evident in reflection character, near 
to target formation as much as possible, and can decrease the effects of incident angle differences on matching results.

\subsection{Quality control of matching processing}

The quality control of purposeless repeated acquisition time-lapse seismic data matching processing mainly includes the following aspects.

1) Initial seismic data analysis. Firstly, the differences of seismic section, time slice, frequency, wavelet, shift of time and energy distribution between the two data sets are analyzed. Then the feasibility of time-lapse seismic match processing is studied. Lastly, taking the high quality data as the reference, the match processing flow is determined.

2) Analysis during matching processing. Whether every step of matching processing reaches the expected target is decided by section comparison before and after matching and difference analysis. It should be noticed that some matching techniques, such as phase correction and matching processing based on normal formation, may cause disorders of the seismic section, so it is necessary to analyze carefully matching results and keep the matching operator stable through proper smoothing, multi-condition optimization and restriction.

3) Matching processing result analysis. The rationality of matching process results should be analyzed by combining with the development status and geological conditions of the real oil field. The differences in normal formation and non-reservoir areas should be smaller in the section, and the range of seismic differences should be within the range of sand body distribution. The unexpected differences should be analyzed carefully from the sides of acquisition and processing, and reasonable and acceptable interpretation should be provided. Fig. 5 and Fig. 6 show the reference data and monitor data, before and after matching processing respectively, in which the reservoir is in the range of $1,300 \mathrm{~ms}$ to $1,500 \mathrm{~ms}$ It demonstrates that the consistency of the two data sets, whose differences are obvious before matching processing, is significantly enhanced by matching processing.

\section{Time-lapse difference calculation}

For a multi-bed hydrocarbon reservoir system, the velocity variation due to production at the upper reservoir bed may cause reflected wave travel time variation in the lower reservoir bed. So the amplitude variations of target formation are induced not only by the changes of reflection coefficients, but by the travel time variation of reflected wave in the lower reservoir bed. To retain amplitude differences caused by reflectivity variations and eliminate those caused by the travel time of reflected wave, the moving window correlation difference method is studied. According to the correlativity of the two seismic data sets calculated by Equation (5) in a small time window, the time shift is determined and applied to the monitor trace before difference calculation.

In Equation (5), $\Phi_{a b}(t)$ is the cross-correlation between the

$$
R(t)=\frac{\sum \Phi_{a b}(t) \times \Phi_{a b}(t)}{\sum \Phi_{a b}(t) \times \Phi_{a b}(t)}
$$
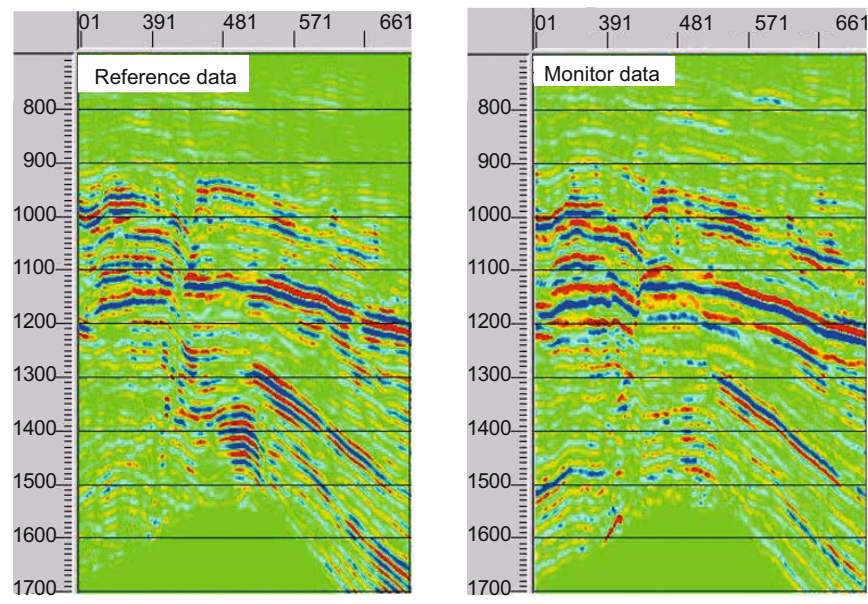

Fig. 5 Comparison of reference and monitor data before matching processing
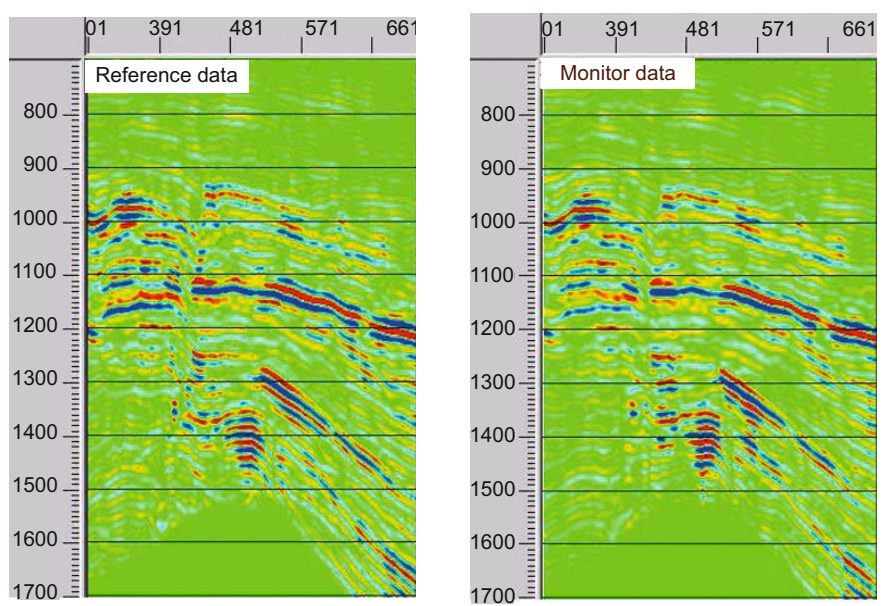

Fig. 6 Comparison of reference and monitor data after matching processing

traces $\mathrm{a}_{t}$ and $\mathrm{b}_{t}$ in the time window from $\mathrm{t} 1$ to $\mathrm{t} 2 . \Phi_{a a}(t)$ and $\Phi_{b b}(t)$ respectively are their autocorrelation in the above window. When $R(t)$ reaches the maximum value, the correlativity of the traces $a_{t}$ and $b_{t}$ is the best, so difference calculation based on correlation can eliminate the amplitude differences caused by the travel time variations and retain those caused by the reflectivity variations.

\section{Conclusions}

This paper studies the processing for purposeless repeated acquisition time-lapse seismic data, providing reference and help for the application of time-lapse seismic technique in China.

1) Pre-processing of time-lapse seismic data must be relative amplitude-preserved processing, which requires not only keeping amplitude, wavelet, frequency spectra stable, but also keeping monitor and reference data processing consistent relatively. Seismic data relative amplitudepreserved processing is one of the key steps.

2) A feasibility study is necessary in the preliminary stage of time-lapse seismic matching processing. During matching 


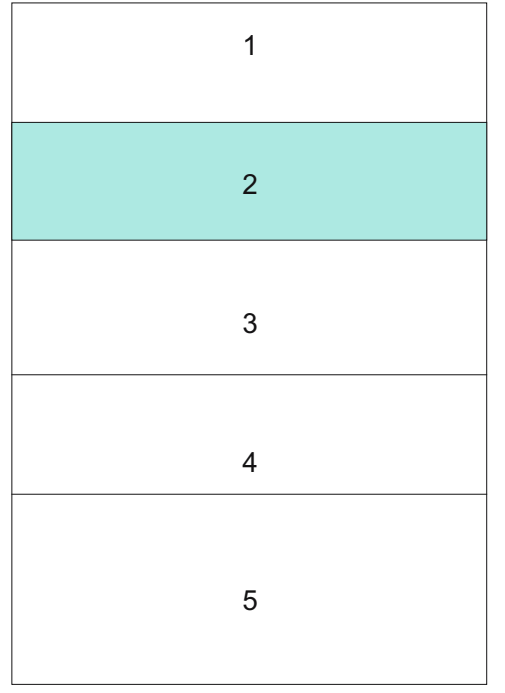

(a)

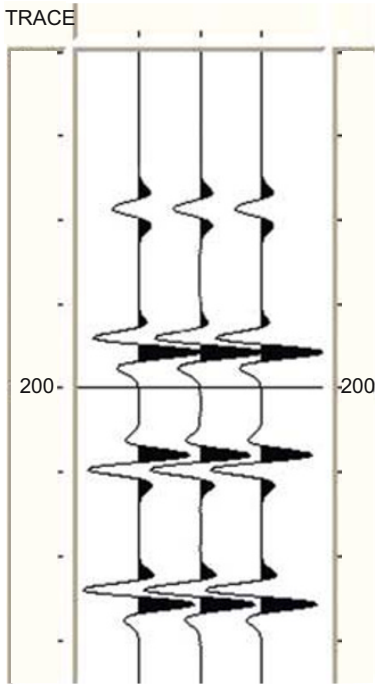

(b)

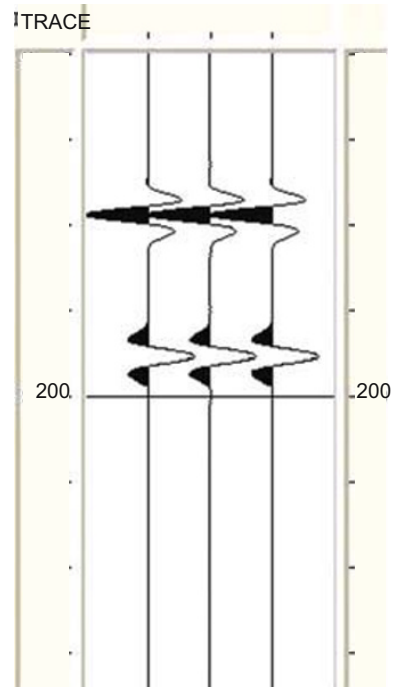

(c)

Fig .7 Comparison of direct difference and moving window correlation difference ( There is velocity variation only in the layer 2 of the geological model before and after production). (a) Geological model (b) Direct difference (c) Difference calculated by moving window correlation difference method

processing, quality control should be conducted to ensure processing purposes, results and stable filter operators.

3) The rationality of time-lapse difference should be determined from the seismic section and time slice by combining with oil field development and geological information.

\section{Acknowledgement}

We thank National Natural Science Function of China (Grant No. 40604016, 40574048) and National 973 Basic Research Program (Grant No. 2007CB209606) for providing financial support to the project.

\section{References}

Andersen C F and van Wijngaarden A. Interpretation of 4D AVO inversion results using rock physics templates and virtual reality visualization, North Sea examples. SEG International Exposition and $77^{\text {th }}$ Annual Meeting. 2007. 2934-2938

Anno P D and Routh P S. A New 4D Workflow for Legacy Seismic Data. SEG International Exposition and $77^{\text {th }}$ Annual Meeting. 2007. 2888-2892

An Y and Wei L C. Differential-equation filtering in seismic data processing. Petroleum Science. 2006. 3(1): 34-38

Chen X H. Normalization for four-dimensional seismic data and its application to real data. Acta Petrolei Sinica. 1999. 20(5): 22-26 (in Chinese)

Gan L D, Yao F C, Zou C, et al. 4-D seismic in water flooding reservoirs: Post-stack cross equalization processing. Progress in Exploration
Geophysics. 2003. 26(1): 54-60 (in Chinese)

Hong T, Sen M K, Stoffa P L, et al. Integrated time-lapse seismic inversion for reservoir petrophysics and fluid flow imaging. SEG International Exposition and $77^{\text {th }}$ Annual Meeting. 2007. 1952-1956

Jin L, Chen X H, Li J Y. A new method for time-lapse seismic matching filter based on error criteria and cyclic iteration. Chinese J. Geophys. 2005. 48(3): 698-703 (in Chinese)

Kloosterman H J and Kelly R S. Successful application of time-lapse seismic data in Shell Expro's Gannet Fields, central North sea UKCS. Petroleum Geoscience. 2003. 9(3): 25-34

Landro M. Discrimination between pressure and fluid saturation changes from time-lapse seismic data. Geophysics. 2001. 66(3): 836-844

Landro M, Veire H H, Duffaut K, et al. Discrimination between pressure and fluid saturation changes from marine multicomponent time-lapse seismic data. Geophysics. 2003. 68(5): 1592-1599

Li J Y and Chen X H. Time-lapse seismic pre-stack attribute analysis by separated wave field numerical modeling. SEG International Exposition and $76^{\text {th }}$ Annual Meeting. 2006. 3270-3273

L i R and $\mathrm{Hu}$ T Y. Cross-equilibration technique in time lapse seismic data processing. Oil Geophysical Prospecting. 2004. 39(4): 425-427 (in Chinese)

Lu X W, Chen X H and Diao S. An approach to time-lapse seismic rebinning and its realization. Progress in Exploration Geophysics. 2004. 27(3): 177-181 (in Chinese)

Wang Z J. Feasibility of time-lapse seismic reservoir monitoring: The physical basis. The Leading Edge. 1997. 16(9): 1327-1329

Yun M H, Yi W Q, Chen W, et al. Seismic monitoring research on water flooding in thin-interbedded reservoir. Oil Geophysical Prospecting. 1999. 34(6): 723-732 (in Chinese)

(Edited by Yang Lei) 